\title{
Triple Immunosuppressive Therapy of Immune- mediated Hemolytic Anemia in a Dog: Case-report
}

\author{
IIldikó BARABÁSI ${ }^{1}$, Alexandra ARION ${ }^{1}$ and Laurenț OGNEAN ${ }^{1 *}$ \\ ${ }^{1}$ University of Agricultural Sciences and Veterinary Medicine, Cluj-Napoca, Romania. \\ Corresponding author: lognean@yahoo.com
}

Bulletin UASVM Veterinary Medicine 72(1) / 2015,

Print ISSN 1843-5270; Electronic ISSN 1843-5378

DOI:10.15835/buasvmcn-vm: 11027

\begin{abstract}
Immune mediated hemolytic anemia (IMHA) is a disease in which the body's own immune system attacks and destroys the erythrocytes causing anemia. This may be primary or secondary as the result of an underlying illness such as neoplasia or intracellular parasites like babesiosis. In most cases the underlying trigger of the anemia may never be found, in which case the disease is called idiopathic immune-mediated anemia.

A double immunosuppressive therapy including prednisolone and cyclosporine as well as various blood transfusions has been administered to the patient, without any results. Mycophenolate mofetil was introduced as the third immunosuppressive drug on the eight day after admission (Park et al., 2012; Wang et al, 2013; West et al, 2014)

The diagnosis of immune-mediated hemolytic anemia has been made after the autoagglutination test of the patient was positive after three RBC washes and negative results for Ehrlichia, Babesia, Leishmania and Borrelia antibodies. Also, tumors have not been found. The patient received in the first eight days of treatment four type specific blood transfusions with erythrocyte concentrate and two immunosuppressive drugs, but still manifested post-transfusion reactions after each transfusion with the hematocrit (Hct) dropping back to initial levels after two days. After the addition of mycophenolate mofetil the Hct stayed stable at a higher level and the patient was discharged two weeks after with a stable Hct of $27 \%$.

Mycophenolate mofetil can be used in severe cases of IMHA when other immunosuppressive agents might not be enough. However due to the frequently encountered side effects it is recommended to be used mostly as a last resort therapeutic agent.
\end{abstract}

Keywords: blood transfusion, IMHA, immunosuppressive therapy, mycopphenolat mophetil, prednisolone.

\section{INTRODUCTION}

Immune mediated hemolytic anemia (IMHA) is one of the most common immune mediated diseases in dogs. The most commonly affected individuals are middle-aged female dogs. Some breed predispositions are also known, with the Cocker Spaniel being one of the most commonly inflicted (Balch and Mackin, 2007).

Hemolysis can be intravascular or extravascular (in the liver or spleen), with the immunopathological process being the same in both cases. Erythrocytes are marked as non-self by the attachment of IgG and IgM and destroyed by macrophages. Red blood cell exrtavascular destruction occures in the liver, but mostly in the spleen and is mediated by IgG, whereas intravascular hemolysis is mainly mediated by IgM (Piek et al., 2009). The degree of autoagglutination and spherocytosis is higher when both immunoglobuline isotypes are involved (Harkin et al., 2012). This mechanism can be initiated by a variety of triggers such as vaccines, neoplasia, certain medication, infectious diseases and other immune mediated illnesses. In this case the hemolytic anemia is secondary to an underlying disease process or an administered substance.

Primary immune mediated hemolytic anemia is rare in veterinary patients, but idiopathic IMHA 
is one of the most common forms of the disease, where the triggering factor is unknown (Piek et al., 2009).

\section{MATERIALS AND METHODS}

A nine year old neutered female mixed breed dog was presented to the emergency service in the Small Animal Internal Medicine department of the Justus-Liebig University in Giessen with lethargy, inapetence and oligodipsy. The symptoms started 4 days earlier with lethargy, weakness and vomiting. A foreign object or poison ingestion could not be excluded by the owner. From the start of the symptoms the patient was treated in a private practice with antipyretic, antiemetic and antibiotic (Metamizol, Emeprid, Cerenia and Enrofloxacin) medication, without any clinical improvement. Hematologic and biochemical findings included: WBC 6.7 G/l, RBC 2.0 T/l, Hb 5.0 g/l, Hct 17\%, absolute reticulocyte count $89.400 \mathrm{MCV} 84 \mathrm{fl}$, PLT $369 \mathrm{G} / \mathrm{l}$, total bilirubin $3.4 \mathrm{mg} / \mathrm{dl}$, total proteins 6.2 $\mathrm{g} / \mathrm{dl}$ and LDH $226 \mathrm{U} / \mathrm{l}$. The blood smear showed a slight anisocytosis and polychromasia. Due to these findings, on the day before presentation in the clinic the patient received a blood transfusion from an unknown donor that did not have any positive effects.

In the clinical examination the dog was anxious but cooperant, with a good general condition, pale and dry mucous membranes with a capillary refill time less than 2 seconds. The pulse was regular and symmetric of medium speed and strength. Pulse rate was $120 /$ minute, respiratory rate $24 /$ minute and rectal temperature $39.3 \mathrm{C}^{0}$. A $2 \times 3 \times 2 \mathrm{~cm}$ large mammary tumor has been found on the left cranial mammary complex. The thorax and abdomen were normal in the physical examination and radiographic imaging. Given the medical history of vomiting unresponsive to treatment, an ultrasound of the abdomen has been performed. Except for a slightly enlarged spleen and gall bladder nothing has been found.

A quick hematological as well as a blood-gas analysis and blood smear has been done in the intensive care unit of the clinic with the following results: RBC $2.03 \mathrm{~T} / \mathrm{l}$, Hct 14.8\%, Hb $4.3 \mathrm{mmol} / \mathrm{l}$, MCV $72.9 \mathrm{fl}$, absolute reticulocyte count 171.1 $\mathrm{G} / \mathrm{l}$, reticulocyte percentage $8.4 \%$, WBC 32.03 G/l, Neutrophiles $19.92 \mathrm{M} / \mathrm{l}$, Lymphocytes 6.38 M/l, Monocytes 5.67 M/l and PLT 156 G/l. Bloodgas analysis results were all within reference range. On the blood smear an anisocytosis and polychromasia with $10-15 \%$ spherocytes and 15$20 \%$ toxic band neutriphiles were observed. An autoagglutination test was performed before the blood transfusion, which resulted in a positive reaction even after the third erythrocyte wash. Together with the spherocytosis seen on the blood smear a preliminary diagnosis of immune mediated hemolytic anemia has been made (Harkin et al., 2012; Hodges and Christopher, 2011).

The patient received a type specific blood transfusion of erythrocyte concentrate in addition to the antithrombotic, anti-inflammatory and gastro-intestinal protection therapy. Immunosuppressive therapy was started with $3.6 \mathrm{mg} / \mathrm{kg}$ prednisolone. During the transfusion the patient developed hyperthermia, a painful abdomen and vomiting. Hematocrit 6 hours posttransfusion was $19 \%$ and the patient improved clinically but developed hematuria. An abdominal ultrasound showed signs of cholecystitis, enteritis and blood cloths in the urinary bladder.

From the third day after admission the prednisolone dose was reduced to $2 \mathrm{mg} / \mathrm{kg}$, but the next day the patient's clinical state deteriorated with RBC dropping to $1.41 \mathrm{~T} / \mathrm{l}$ and the Hct to $12 \%$. A blood transfusion of $270 \mathrm{ml}$ type specific erythrocyte concentrate and cyclosporine as a second immunosuppressive medicine has been started at a dose of $5.3 \mathrm{mg} / \mathrm{kg}$. Transfusion reactions have been observed this time as well and 8 hours post-transfusion the patient developed signs of hemolysis, but improved clinically. The Hct and RBC remained at pre-transfusion levels. Another type specific erythrocyte concentrate was given that raised the Hct to $22 \% 6$ hours after transfusion.

Three days after the last transfusion RBC dropped to $1.18 \mathrm{~T} / \mathrm{l}$ and Hct to $12 \%$ making the administration of another type specific erythrocyte concentrate necessary. Post-transfusion reactions haven't been as strong this time, but the disease progression justified the introduction of a third immunosuppressive drug (Wang et al., 2014; Park et al., 2012). From the eighth day after admission mycophenolat mofetil has been added to the treatment protocol in a dose of $10 \mathrm{mg} / \mathrm{kg}$.

After the initiation of the triple immunosuppressive therapy, the patient improved every day clinically, it's appetite came back, urine and stool were normal. Hematocrit and RBC levels 
rose slowly over the next week. As the patients gastro-intestinal symptoms ceased, the therapy was changed to oral administration.

Due to the progressive clinical improvement and stable hematological parameters, the patient was discharged after 18 days with a Hct of $27 \%$. The triple immunosuppressive therapy, along with the antithrombotic and gastrointestinal protective medication was given for another week as an outpatient treatment, until the next control examination.

\section{RESULTS AND DISCUSSIONS}

Canine IMHA patients often require the use of more than one immunosuppressive medication as treatment can last for several months and combination of these drugs allows the physician to use a lower dose of each one, thus reducing the side-effects (Whitley and Day, 2011).

Still, a triple immunosuppressive therapy is used quite rarely in veterinary medicine, but the severity of the hemolysis in this particular case and the frequent relapses to a critical state of the patient, justified the usage of all three drugs. Treatment has been initiated with a glucocorticoid drug with the later addition of cyclosporine and mycophenolate mofetil, to the treatment protocol until the desired effect has been reached (Tab. ).

The patient was closely monitored after discharge, with follow-up hematological tests every 2 weeks. At the time of the first followup control the patient has been under a triple immunosuppressive therapy for 24 days. Hematological parameters showed a very good recovery with $\mathrm{RBC}$ at $4.74 \mathrm{~T} / \mathrm{l}$, Hct $38 \%$ and spherocytosis bellow 4\%. Due to this evolution the prednisolone dose has been reduced by $25 \%$ and mycofenolat mofetil was taken out entirely. The first two follow-up examinations were conducted at 2 weeks apart, after which the patient was called in every 4 weeks for the following 3 months.

The owner reported severe glucocorticoid induced side-effects on the first follow-up visit, manifested thru massive polyuria/polydipsia and polyphagia.

Hematologic parameters steadily increased at each follow-up control, allowing the reduction by $25 \%$ of the prednisolone dose. Side-effects produced by the glucocorticoid medication slowly reduced as the dose was lowered.

On the last recorded visit the patient's hematological parameters were all within normal reference range, RBC 6.11 T/l, Hct 40\% and spherocytosis $0 \%$. The immunosuppressive therapy has been reduced at this point to 0.5 $\mathrm{mg} / \mathrm{kg}$ prednisolone and cyclosporine remained unchanged at $5.3 \mathrm{mg} / \mathrm{kg}$ (Tab. 2)

Further follow-up examinations were done in a private practice from which the only feedback given was that the patient is doing very well clinically, the hematologic parameters are within normal reference range and any signs of glucocorticoid induced side-effects have ceased.

Mycofenolate mofetilisanimmunosuppressive medication used mostly in human medicine on transplant patients. The immunomodulating effect of the drug consists in the prevention of $\mathrm{T}$ and $\mathrm{B}$ cell proliferation and differentiation by reversible inhibition of the key enzyme in the de novo synthesis of purine (Whitley and Day, 2011). Side effects of the medication include mostly gastro-intestinal symptoms.

Published data on the use of mycofenolate mofetil in veterinary medicine is contradictory, some authors reporting severe gastrointestinal symptoms in canine patients that had to lead

Tab. 1. RBC, Hct and spherocytosis evolution under the immunosuppressive therapy during hospitalization

\begin{tabular}{ccccccc}
\hline \multirow{2}{*}{$\begin{array}{c}\text { Hematological } \\
\text { parameters }\end{array}$} & $3.6 \mathrm{mg} / \mathrm{kg}$ prednisolone & \multicolumn{2}{c}{$\begin{array}{c}2 \mathrm{mg} / \mathrm{kg} \text { prednisolone }+ \\
5.3 \mathrm{mg} / \mathrm{kg} \text { cyclosporine }\end{array}$} & $\begin{array}{c}2 \mathrm{mg} / \mathrm{kg} \text { prednisolone }+ \\
5.3 \mathrm{mg} / \mathrm{kg} \text { cyclosporine }+ \\
10 \mathrm{mg} / \mathrm{kg} \text { mycofenolat mofetil }\end{array}$ \\
\cline { 2 - 7 } & & & Day 3 & Day 7 & Day 10 & Day 15 \\
\hline RBC (T/l) & 2.03 & 2.66 & 1.41 & 1.18 & 1.86 & 2.73 \\
\hline Hct (\%) & 14.8 & 19 & 12 & 12 & 19 & 27 \\
\hline Spherocytosis (\%) & $5-10$ & $40-50$ & 4 & $5-10$ & $5-10$ & $<5$ \\
\hline
\end{tabular}


Tab. 2. Evolution of hematological parameters after discharge

\begin{tabular}{|c|c|c|c|}
\hline $\begin{array}{c}\text { Hematological } \\
\text { parameters }\end{array}$ & $\begin{array}{c}2 \mathrm{mg} / \mathrm{kg} \\
\text { prednisolone }+ \\
5.3 \mathrm{mg} / \mathrm{kg} \\
\text { cyclosporine }+ \\
10 \mathrm{mg} / \mathrm{kg} \\
\text { mycofenolat mofetil }\end{array}$ & $\begin{array}{cc}1.6 \mathrm{mg} / \mathrm{kg} & 1.3 \mathrm{mg} / \mathrm{kg} \\
\text { prednisolone }+ & \text { prednisolone }+ \\
5.3 \mathrm{mg} / \mathrm{kg} \text { cyclosporine } 5.3 \mathrm{mg} / \mathrm{kg} \text { cyclosporine } 5 .\end{array}$ & $\begin{array}{c}1 \mathrm{mg} / \mathrm{kg} \\
\text { prednisolone }+ \\
3 \mathrm{mg} / \mathrm{kg} \text { cyclosporine }\end{array}$ \\
\hline & $\begin{array}{c}14 \text { days after } \\
\text { discharge }\end{array}$ & 26 days after discharge 55 days after discharge 80 & 6 days after discharge \\
\hline $\mathrm{RBC}(\mathrm{T} / \mathrm{l})$ & 4.74 & 4.86 & 6.11 \\
\hline Hct (\%) & 38 & 38 & 40 \\
\hline Spherocytosis (\%) & $<4$ & $<4$ & 0 \\
\hline
\end{tabular}

to the drugs discontinuation from the therapy (West and Hart, 2014), while other researchers encountered no or very mild side-effects and very good therapeutic results. (Wang et al., 2013, Park et al., 2012)

In this particular case clinical and hematological improvement of the patient has been observed only after the introduction of mycofenolate mofetil in the therapeutic protocol. Severe hemolysis has been observed with the use for the first three days of treatment with only prednisolone in an immunosuppressive dose of $3.6 \mathrm{mg} / \mathrm{kg}$ as well as the following 5 days when cyclosporine was also added.

The patient was already manifesting gastrointestinal symptoms on the day of presentation. These were later attributed to the enteritis and cholecystitis suspected in the abdominal ultrasound examination. The gastro-intestinal symptoms persisted though the first eight days of hospitalization and began to fade only after the overall clinical improvement and stabilization of the hematological parameters of the patient on the triple immunosuppressive treatment protocol.

\section{CONCLUSIONS}

Immune mediated hemolytic anemia in dogs is a difficult to treat disease that may require the usage of multiple immunosuppressive medications. The patient required multiple blood transfusions during its hospital stay and although every erythrocyte concentrate has been type specific, it triggered immediate transfusion reactions. Setbacks in form of hemolysis were observed after every blood transfusion, except the last one, when the third immunosuppressive medication, mycofenolate mofetil has been introduced.

The patient did not show any symptoms that could have been related to the potential toxic effects of mycofenolate mofetil manifested mostly through gastro-intestinal symptoms. Given the fact that the patient had a colecystitis and enteritis diagnosed via ultrasound, these did not worsen after the addition of the mycofenolate mofetil.

Clinical as well as hematological parameter improvement and stabilization occurred in a relatively short time, the patient recovered well enough to be released after 10 days on a triple immunosuppressive treatment.

Glucocorticoidinduced symptoms represented by polyuria/polydipsia and polyphagia persisted for another 86 days after discharge, when the prednisolone dose could be safely reduced to less than $1 \mathrm{mg} / \mathrm{kg}$. Severity of these symptoms gradually reduced as the prednisolone dose has been steadily lowered.

\section{REFERENCES}

1. Balch A., and Mackin A.(2007).Canine immune-mediated hemolytic anemia: Pathophysiology, clinical signs and diagnosis. Compendium on continuing education for the practicing veterinarian. 29(4):217-225.

2. Harkin KR., Hicks JA. and Wilkerson MJ. (2012). Erythrocyte-bound immunoglobulin isotopes in dogs with immune-mediated hemolytic anemia: 54 cases (2001-2010).Journal of the American Veterinary Medical Association 241(2):227-232.

3. Hodges J., and Christopher MM. (2011). Diagnostic accuracy using erythrocyte indices and polychromasia to indetify regenerative anemia in dogs. Journal of the American Veterinary Medical Association. 238(11):14521458. 
4. Park HJ., Lee DY., Song KH. (2012). Application of mycophenolate mofetil for immune-mediated hemolytic anemia in two dogs. Korean J Vet Res 52(3):209-211.

5. Piek CJ., Junius G., Dekker A., Schrauwen E., Slappendel RJ. and Teske E. (2008). Idiopathic Immune-Mediated Hemolytic Anemia: Treatment Outcome and Prognostic Factors in 149 Dogs. Journal of Veterinary Internal Medicine 22:366-373.

6. Wang A., Smith JR., Creevy KE. (2013). Treatment of canine idiopathic immune-mediated hemolytic anemia with mycophenolate mofetil and glucocorticoids: 30 cases (2007 to 2011). Journal of Small Animal Practice. 54(8):399-404.

7. West LD. and Hart JR. (2014). Treatment of idiopathic immune-mediated hemolytic anemia with mycophenolate mofetil in five dogs.Journal of Veterinary Emergency and Critical Care 24(2):226-231.

8. Whitley NT. and Day MJ. (2011). Immunomodulatory drugs and their application to the management of canine immune-mediated disease. Journal of Small Animal Practice. 52:70-85. 